\title{
A review of solifenacin in the treatment of urinary incontinence
}

\author{
Ramandeep Basra \\ Con Kelleher \\ Department of Gynaecology, Guys \\ and St Thomas NHS Foundation Trust, \\ London, England, UK
}

\begin{abstract}
Overactive bladder $(\mathrm{OAB})$ is a prevalent condition which has an adverse effect on quality of life. The presence of urgency incontinence confers significant morbidity above and beyond that of $\mathrm{OAB}$ sufferers who are continent. The primary treatment for $\mathrm{OAB}$ and urgency incontinence is a combination of behavioral measures and antimuscarinic drug therapy. The ideal antimuscarinic agent should effectively relieve the symptoms of $\mathrm{OAB}$, with the minimum of side effects; it should be available as a once-daily sustained release formulation and in dosage strength that allows easy dose titration for the majority of sufferers. Solifenacin succinate was launched in 2005, and has been shown in both short and long term clinical trials to fulfill these requirements. Solifenacin is a competitive M3 receptor antagonist with a long half-life (45-68 hours). It is available in two dosage strengths namely a 5 or $10 \mathrm{mg}$ once-daily tablet. The efficacy and tolerability of solifenacin for the treatment of all symptoms of OAB has been evaluated in a number of large, placebo controlled, randomized trials. Long-term safety, efficacy, tolerability and persistence with treatment have been established in an open label 40 week continuation study.
\end{abstract}

Keywords: solifenacin, urinary incontinence, overactive bladder

\section{Introduction}

Urinary incontinence has been defined by the International Continence Society as "the complaint of any involuntary leakage of urine" (Abrams et al 2000). The prevalence of urinary incontinence has been estimated as $13.1 \%$ in women and $5.4 \%$ in men in a recent population-based survey conducted across 5 countries (Irwin et al 2006). Overactive bladder $(\mathrm{OAB})$ is a symptom complex characterized by urinary urgency, which in turn drives the symptoms of frequency and nocturia with or without urgency incontinence. Epidemiological studies (Irwin et al 2006) have estimated the prevalence of $\mathrm{OAB}$ to be $14 \%-16 \%$ after the age of 40 years and prevalence increases inexorably with age for both men and women. It is estimated that one third of OAB patients have urgency incontinence. The presence of urgency incontinence confers significant morbidity above and beyond that of OAB sufferers who are continent (Milsom et al 2001; Stewart et al 2003).

The primary treatment for $\mathrm{OAB}$ and urgency incontinence is a combination of behavioral measures (including bladder retraining) and antimuscarinic drug therapy. Despite the proven efficacy of these drugs for the relief of OAB symptoms, side effects impair tolerability and persistence with therapy, dosing regimens are not always simple, efficacy has been questioned, formulary listed dosage strengths are not always optimal and their cost may be prohibitive.

The ideal antimuscarinic agent should effectively relieve the symptoms of OAB, with the minimum of side effects; it should be available as a once daily sustained release formulation and in dosage strength that allows easy dose titration for the majority of sufferers. Solifenacin succinate is a relatively new antimuscarinic which 
has been shown in both short and long term clinical trials to fulfill these requirements (Chapple 2000).

The aim of this review is to describe the pharmacological properties, and clinical evidence for the use of solifenacin.

\section{Pathology of overactive bladder}

The etiology of OAB is multifactorial. Although a large proportion of cases are idiopathic, recognized contributing factors include lower urinary tract pathology (infection, calculi, and stones), neurological conditions (stroke, dementias, multiple sclerosis), systemic conditions (congestive heart failure, diabetes mellitus), functional and behavioral conditions (caffeine and alcohol consumption, mobility), and side effects of medication (Ouslander 2004).

Involuntary detrusor muscle contractions may be associated with OAB. Bladder contraction is mediated by acetylcholine; a peripheral neurotransmitter which acts on muscarinic receptors of the detrusor muscle. Of the five known muscarinic receptor subtypes (M1-M5), M2 and M3 receptors are found in the bladder and M3 receptors appears to be the most important for bladder contractility. The true function of M2 receptors within the bladder is incompletely understood but it is thought that they may oppose sympathetically mediated smooth muscle relaxation or result in the activation of a non-specific cationic channel and activation of potassium channels (Hedge et al 1997, 1999). It is also thought that in certain disease states such as neurogenic bladder dysfunction the $\mathrm{M} 2$ receptors may become more important in mediating detrusor contractions (Braverman et al 1998).

\section{Antimuscarinic agents}

Older antimuscarinic agents such as oxybutynin are neither bladder nor muscarinic receptor subtype selective and are thus associated with greater generalized anticholinergic side effects. Commonly reported side effects of all antimuscarinic drugs include dry mouth, constipation, blurred vision, gastroesophageal reflux, and cognitive impairment. Adverse events and multiple daily dosing regimens affect drug tolerability, compliance, and ultimately treatment efficacy. A study in 1997 examining the real world (as opposed to clinical trial) efficacy of predominantly immediate release oxybutynin demonstrated a significant impairment of treatment efficacy due to antimuscarinic side effects. Many patients who felt the medication was effective were unable to tolerate side effects and consequently only $18 \%$ of patients remained on treatment at six months (Kelleher et al 1997a). The slow release preparation of oxybutynin has been shown to be as efficacious as immediate release oxybutynin, however is associated with a much reduced side effect profile. Controlled release preparations are associated with reduced peak serum drug and metabolite levels, which lead to a reduced incidence and severity of adverse events (Birns et al 1997).

Recently concern has arisen regarding the potential for significant cognitive impairment of patients receiving antimuscarinic drugs which exert an effect on M1 receptors and which can cross the blood brain barrier. Kay et al (2006) have shown using name face recognition testing that at a dose of $20 \mathrm{mg}$ once daily oxybutynin has an effect similar to that of 10-20 years of cognitive deterioration. This is of major importance amongst elderly patients who may already exhibit a degree of cognitive impairment and for whom OAB is most prevalent. It is clearly also important among this group of patients who are often on multiple other medications some of which have anticholinergic effects. Kay et al showed that darifenacin which is highly M3 selective and tolterodine which does not cross the blood brain barrier to any significant degree do not affect cognitive function. It is likely that solifenacin will in similar studies for the same reasons have no effect on cognitive function, and to date cognitive impairment has not been reported with this drug in clinical trials or clinical practice (Kay et al 2006).

Since the voluntary withdrawal of terodiline from the market by Pharmacia in 1993 due to concerns regarding cardiac function, in particular the occurrence of Torsades de Pointes in susceptible individuals cardiac function assessment has been an integral part of the safety profile of these drugs. None of the currently prescribed antimuscarinics used for the treatment of OAB have proven adverse cardiac profiles despite vigorous testing (Monahan et al 1990).

\section{Pharmacokinetics of solifenacin}

In drug development solifenacin has been shown to have functional selectivity for the bladder over other organs in animal models. In vivo and in vitro studies have shown greater bladder selectivity over salivary gland tissue than tolterodine and oxybutynin. This relative bladder selectivity was the rationale for development of solifenacin in the treatment of OAB (Hatanaka et al 2003; Ohtake et al 2003).

Solifenacin is a competitive M3 receptor antagonist. Peak plasma levels are reached 3-8 hours following oral administration. Solifenacin has $98 \%$ plasma protein binding and is highly distributed to peripheral tissues. Solifenacin has $90 \%$ bioavailability and a long half-life of 45-68 hours. The major metabolite has been found to have similar binding profile to muscarinic receptors as that of solifenacin but less potency (Hedge et al 1997). 
Other animal studies have shown that the metabolism of solifenacin is mediated by the liver enzyme CYP3A4 and major metabolites can be detected in the urine feces and bile (Onderwater 1999). Solifenacin at therapeutic dosage does not appear to inhibit liver cytochrome $\mathrm{P} 450$ isoenzymes and therefore is unlikely to cause pharmacokinetic drug-drug interactions based on CYP dependent metabolism of co-administered drugs. Solifenacin is not licensed for use in pediatric or pregnant patients (Yamanouchi Pharma America 2004).

\section{Clinical efficacy}

Solifenacin is available in 2 dosage strengths namely a 5 or $10 \mathrm{mg}$ once-daily tablet which can be taken with or without food. Phase 3 studies demonstrated a dose-dependent effect in the relief of the common symptoms of OAB namely frequency, urgency, nocturia and urgency incontinence. Patients are normally commenced on $5 \mathrm{mg}$ and titrated according to clinical need and personal preference to $10 \mathrm{mg}$ after 4-6 weeks of treatment. In clinical trials approximately $50 \%$ of patients request the higher dose when offered the opportunity to do so (Haab et al 2005). Side effects are also dose dependent, and include dry mouth, constipation, and blurred vision.

It is important to remember that in the original 12-week phase 3 studies upon which initial efficacy and tolerability data were described, patients were randomized without choice to either the $5 \mathrm{mg}$ or $10 \mathrm{mg}$ dosage. Ultimately from our present understanding of dose selection this would have resulted in $50 \%$ of patients receiving too high or too low a dosage for their requirements, and both efficacy and tolerability would be influenced by this process. Ultimately in the 40-week extension study, patients were all recommenced on $5 \mathrm{mg}$ solifenacin for the first 4 weeks of open label treatment after completion of the original 12-week studies and 4 weeks thereafter offered the option of dose escalation. At this time $50 \%$ of patients increased their dosage with improvement in efficacy without noticeable tolerability issues (Yamanouchi Pharma America 2004).

\section{Clinical trials}

There have been several large, randomized clinical trials evaluating the efficacy, safety, tolerability, and persistence with solifenacin (Table 1). Each of these trials will be discussed.

Chapple et al (2004a) conducted a phase 2 study to evaluate the effective dose of solifenacin for the treatment of OAB. This was a multicenter, double blind, placebo and tolterodine controlled trial. Following a single blind 2-week placebo run in, patients were randomized to 4 weeks of either placebo, solifenacin (2.5 mg, $5 \mathrm{mg}, 10 \mathrm{mg}$, or $20 \mathrm{mg}$ doses $)$ or twice-daily immediate-release tolterodine $(2 \mathrm{mg}) .265$ patients were enrolled and 192 patients completed the study.

The primary outcome measure of the study was the change from baseline, in the mean number of voids per 24 hours. Secondary outcome measures included the change in volume of urine voided per void, and the mean number of urgency and incontinence episodes per 24 hours. Quality of life changes were assessed using the 27 item Contilife questionnaire.

Results of the study showed a significant reduction in the mean number of voids per 24 hours, and an increase in the mean volume voided per void in the study group taking $5 \mathrm{mg}, 10 \mathrm{mg}$, and $20 \mathrm{~g}$ of solifenacin, when compared with the placebo group. There was no statistically significant reduction in voiding frequency, and volume voided per void in the tolterodine and $2.5 \mathrm{mg}$ solifenacin treated patients compared with placebo (Figure 1).

The efficacy and tolerability of solifenacin was found to be dose dependant, with the greatest reduction in frequency occurring with the $20 \mathrm{mg}$ dose. Neither solifenacin nor tolterodine treated patients experienced a significant reduction in the number of urgency or incontinence episodes. Quality of life assessment using the Contilife questionnaire showed an improvement in 4 domains (daily life activities, emotional consequences, sexuality, and self-image) in the solifenacin treated patients, compared with improvement in the daily life activities domain in the tolterodine treated group, when compared with placebo.

Dry mouth and constipation were the most commonly reported adverse events. The incidence of dry mouth was greatest in the solifenacin $20 \mathrm{mg}$ treated group $(\mathrm{n}=14,38 \%)$, followed by the tolterodine treated group $(n=9,24 \%)$, and then the 5 and $10 \mathrm{mg}$ dose treated groups $(n=5,14 \%$ for both groups).

Patients taking $20 \mathrm{mg}$ of solifenacin reported the highest incidence of adverse events, followed by those patients taking tolterodine. The lowest incidence of adverse events was reported by patients taking 2.5 and $5 \mathrm{mg}$ solifenacin.

Fourteen patients (7\%) discontinued from the study due to adverse events; 7 of these patients were in the $20 \mathrm{mg}$ solifenacin-treated group (one subject dropped out during the placebo run-in period), 6 patients were in the $10 \mathrm{mg}$ solifenacin group, and 1 patient was taking $5 \mathrm{mg}$ of solifenacin.

There have been 4 phase 3 trials, a 1-year open label extension study and a solifenacin and tolterodine head-tohead study assessing the safety and tolerability of solifenacin in patients with $\mathrm{OAB}$. 


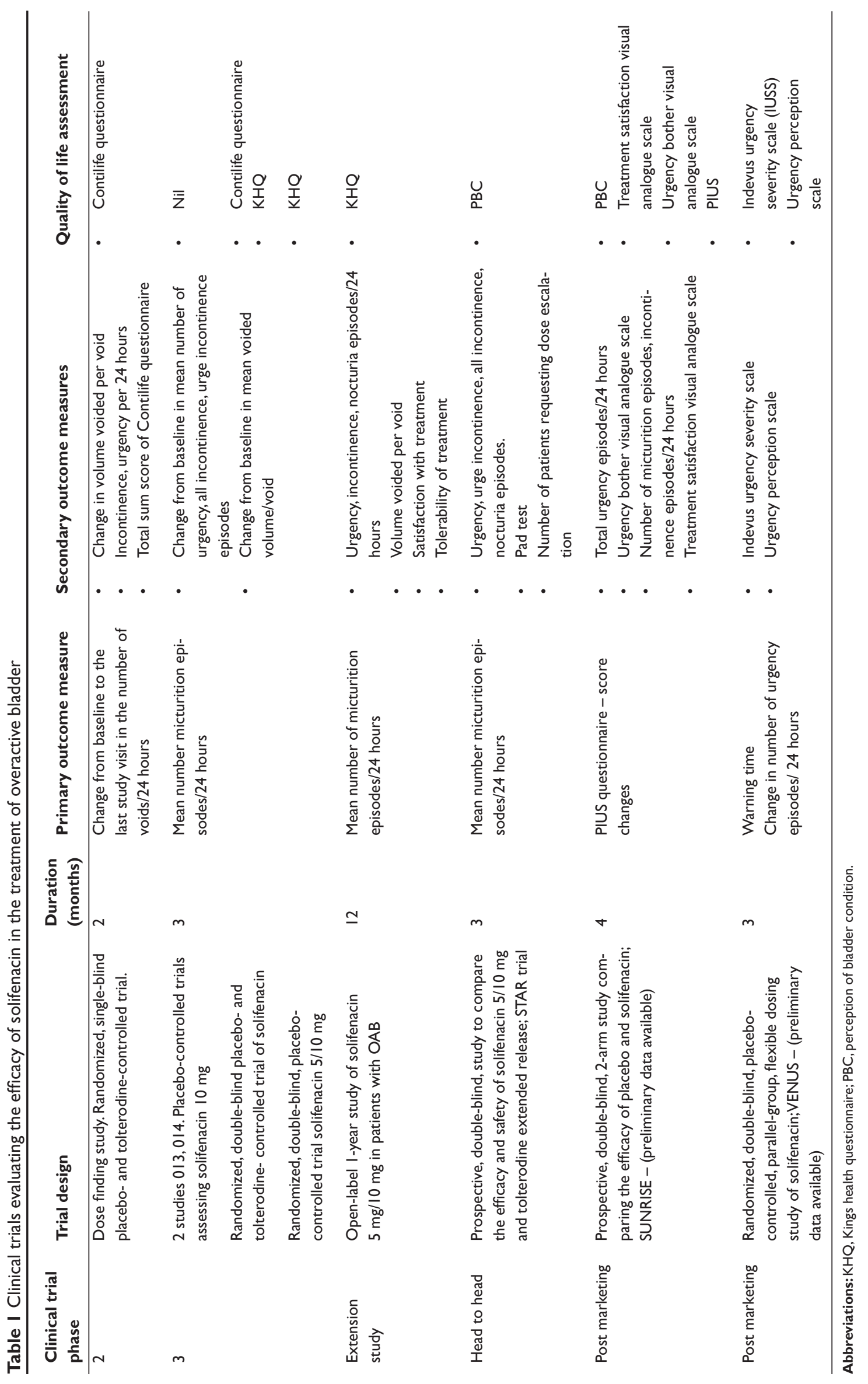




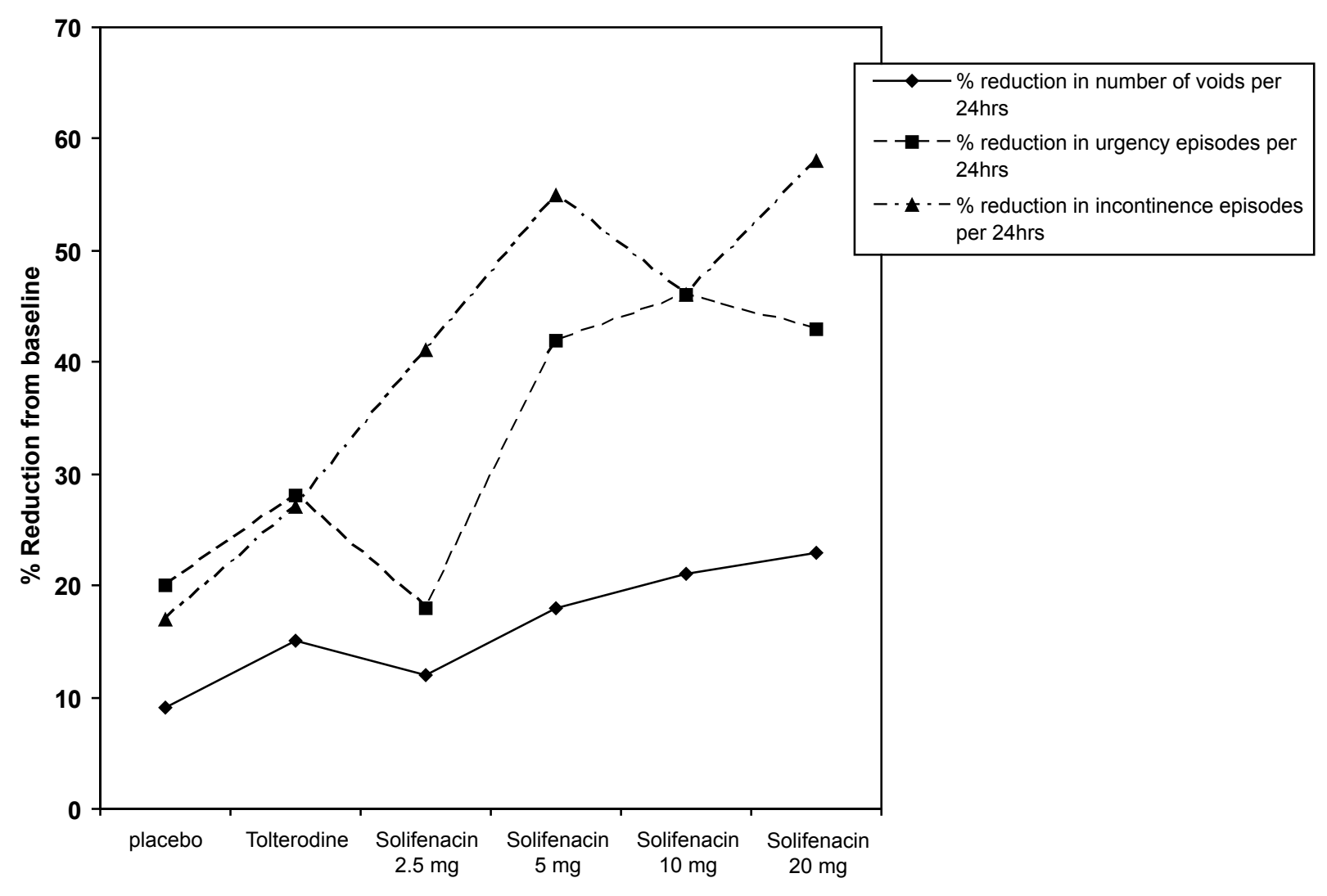

Figure I Changes in bladder diary variables in a phase 2, solifenacin dose finding study.

Two initial phase 3 clinical trials were designed to evaluate the efficacy and safety of $10 \mathrm{mg}$ solifenacin in double blind, placebo-controlled trials (studies 013 and 014). The primary endpoint for both studies was the change from baseline to 12 weeks in the number of micturitions in 24 hours. The mean change in the number of incontinence episodes per 24 hours and mean volume voided per micturition, were used as secondary endpoints. Both studies showed solifenacin $10 \mathrm{mg}$ to be superior to placebo in reducing micturition frequency, incontinence and urgency episodes, and the volume voided per micturition episode. There was no statistically significant reduction in nocturia episodes in both studies. 1208 patients were recruited into the studies. Pharmacokinetic studies were performed before drug dosing, at steady state and post-dosing. Both studies reported mild to moderate adverse events, the most commonly reported being dry mouth and constipation. In study 013 there was one discontinuation due to dry mouth.

Cardozo et al (2004) conducted a 12-week, multicenter, randomized, double blind placebo controlled trial assessing the efficacy of 5 and $10 \mathrm{mg}$ doses of solifenacin. Safety and tolerability of solifenacin were secondary study objectives.
Patients were randomized to once daily doses of placebo, $5 \mathrm{mg}$ or $10 \mathrm{mg}$ of solifenacin.

The primary endpoint for this 12-week trial was the mean change in micturition episodes in 24 hours. Changes from baseline in the mean umber of urgency, nocturia and incontinence episodes, and the mean volume voided per void, were used as secondary endpoints. 907 patients took part in the trial, and data from 857 patients was analyzed. 281 patients received placebo, 286 received $5 \mathrm{mg}$ solifenacin and placebo, and 290 received $10 \mathrm{mg}$ of solifenacin.

The mean reductions in the number of micturition, urgency, and nocturia episodes in 24 hours are shown in Figure 2. The reductions in urinary frequency and urgency episodes with solifenacin treatment were statistically significant for both doses of solifenacin. One of the major findings of this study was the significant reduction in nocturia episodes in patients taking a $10 \mathrm{mg}$ dose of solifenacin.

Half of the patients who were incontinent at baseline and received treatment with solifenacin $(5 \mathrm{mg}$ and $10 \mathrm{mg}$ ) were dry at the end of the study period. Rates of dry mouth were $7.7 \%, 23 \%$, and $2.3 \%$ for solifenacin $5 \mathrm{mg}, 10 \mathrm{mg}$ and placebo respectively. 29 patients $(3.2 \%)$ withdrew from 


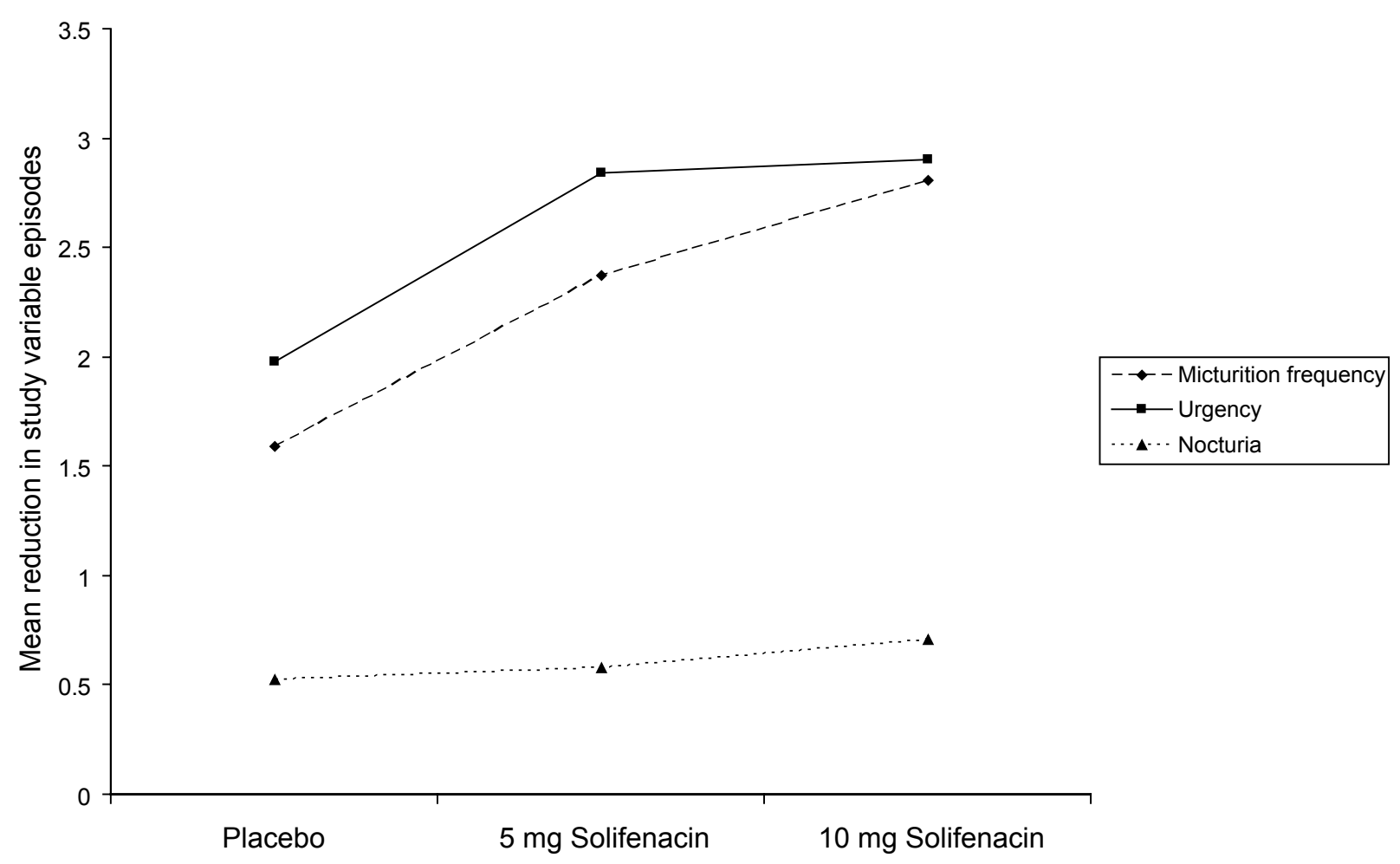

Figure 2 Reduction from baseline in the number of micturition and urgency episodes in 24 hours, and episodes of nocturia in patients receiving placebo, $5 \mathrm{mg}$, or $10 \mathrm{mg}$ of solifenacin in a phase 3 trial (drawn from data of Cardozo et al 2004).

the study due to adverse events; this was greatest in the solifenacin $10 \mathrm{mg}$ treated group $(\mathrm{n}=12,3.9 \%)$, then the placebo group $(n=10,3.3 \%)$. Seven patients $(2.3 \%)$ in the $5 \mathrm{mg}$ solifenacin-treated group withdrew from the study due to adverse events.

A 12-week phase 3a study assessing the efficacy of $5 \mathrm{mg}$ and $10 \mathrm{mg}$ solifenacin was conducted in a multicenter, double-blind, placebo-controlled trial, using $2 \mathrm{mg}$ of immediate release tolterodine as an active comparator (Chapple et al 2004b). The primary aim was to assess the efficacy of solifenacin 5 and $10 \mathrm{mg}$ whilst the secondary aims were to compare the safety and efficacy with that of $2 \mathrm{mg}$ immediate release (IR) tolterodine. After a 2-week placebo run-in period, subjects were randomized to either $2 \mathrm{mg}$ IR tolterodine twice daily, placebo, solifenacin $5 \mathrm{mg}$ or $10 \mathrm{mg}$. Baseline to end of study changes in the mean number of urgency, mixed incontinence and urgency incontinence episodes were used as study outcome measures. In addition, the mean number of voids per 24 hours and mean volume voided per void were also assessed.

1077 men and women received one of the study drugs. Results of the study showed a statistically significant reduction in the number of urgency and urgency incontinence episodes in the patients receiving solifenacin, when compared with placebo. These changes were not statistically significant in the tolterodine-treated group when compared with placebo. There was a significant reduction in urinary frequency with all active treatments, this effect being greatest in the solifenacin $10 \mathrm{mg}$, and then the $5 \mathrm{mg}$ group. There was a statistically significant improvement in volume of urine per void in the solifenacin- and tolterodine-treated groups, when compared with placebo. The changes in study outcome measures are shown in Figure 3.

$2.9 \%$ of participants $(n=31)$ withdrew from the study due to adverse events, with the highest rate of discontinuation in the placebo-treated group $(n=10,3.7 \%)$. Five $(1.9 \%)$ patients taking tolterodine and 16 patients taking solifenacin $(n=9$, $3.2 \%$ taking $5 \mathrm{mg}$ dose, $\mathrm{n}=7$ taking $10 \mathrm{mg}$ dose) withdrew from the study due to adverse events.

The incidence of dry mouth was $14 \%, 21.3 \%$, and $18.6 \%$ in the $5 \mathrm{mg}$ and $10 \mathrm{mg}$ solifenacin- and tolterodine-treated patients respectively. Constipation was reported in a greater number of patients receiving solifenacin than tolterodine.

The study found the 5 and $10 \mathrm{mg}$ doses of solifenacin to be more effective than placebo, in the treatment of OAB.

These preliminary studies lead to the STAR study; a head to head clinical trial comparing the efficacy of the existing market leader tolterodine extended release with dose flexible 


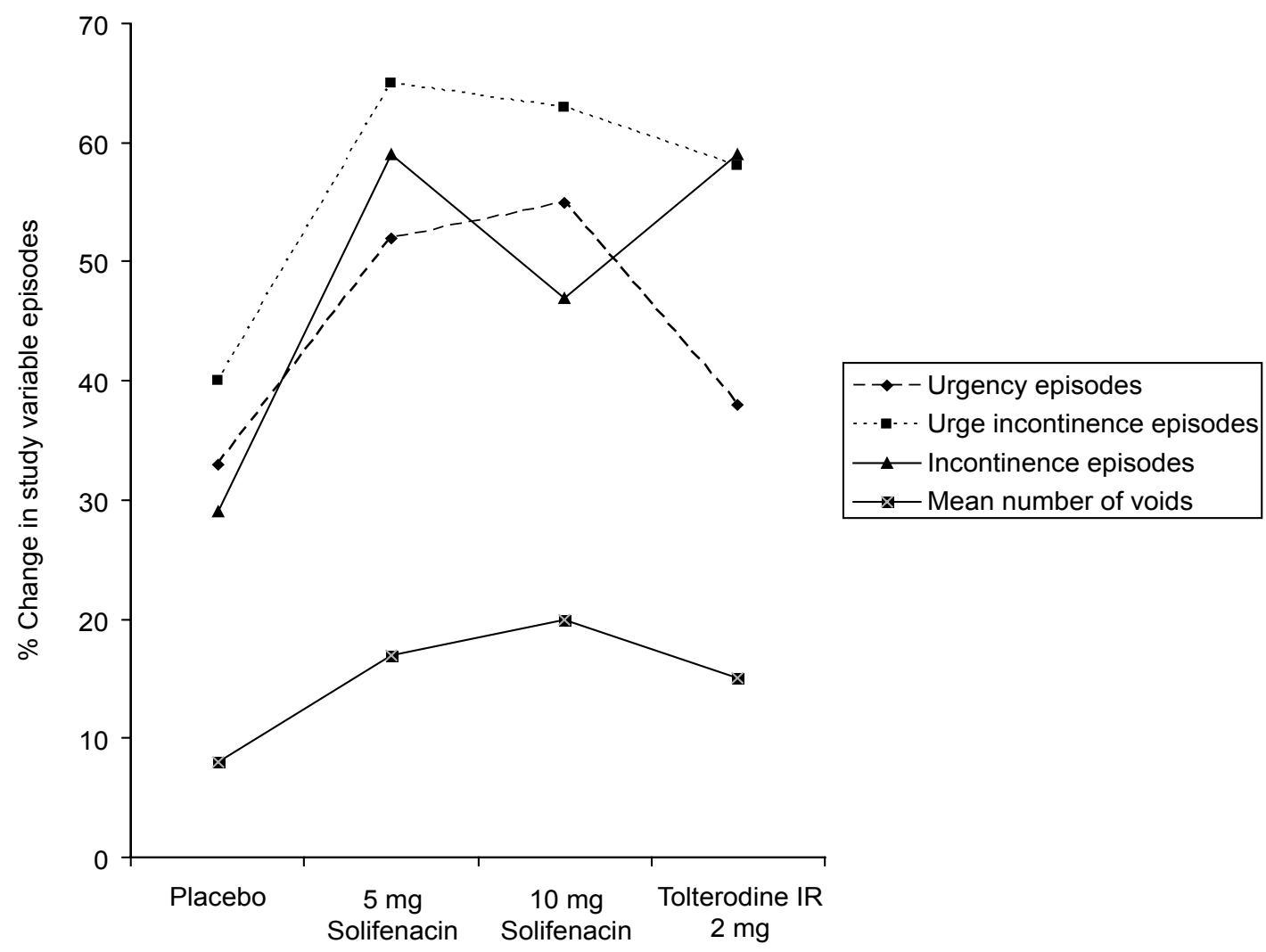

Figure 3 The percentage change from baseline in the mean number of urgency, incontinence, and urge incontinence episodes and mean voids in a 24-hour period (Drawn from data of Chapple et al 2004b).

solifenacin. This was a prospective, double blind, 2 arm, parallel group, 12-week study (Chapple et al 2005). 1200 subjects were randomized, 593 were commenced on $5 \mathrm{mg}$ solifenacin and 607 on $4 \mathrm{mg}$ extended release tolterodine. The study design is described in Figure 4.

In this type of study the initial analysis is one of noninferiority of the test drug, and once established assessment of superiority may follow. The results of the study showed that solifenacin was not inferior to tolterodine in reducing the number of voids per 24 hours; 2.45 and 2.24 episodes, respectively. Solifenacin treatment resulted in a significant improvement in urgency, nocturia, urge incontinence, and overall incontinence when compared with tolterodine. Reduction in pad usage was significantly greater in the solifenacin-treated group than the tolterodine-treated group (1.72 and 1.19 respectively). Four weeks after treatment initiation, $48 \%$ of patients on solifenacin and $51 \%$ on tolterodine requested a dose increase.

The perception of bladder condition questionnaire (PBC) is a validated single-item questionnaire, which asks people to choose 1 of 6 responses describing the severity of their bladder condition. Assessment of the PBC questionnaire scores showed a greater improvement in the solifenacin-treated group than the tolterodine-treated group (1.51 and 1.33 respectively).

Adverse events were those associated with antimuscarinic therapy and were mild to moderate in severity.

The study concluded that the flexible dosing of solifenacin is more effective in treating $\mathrm{OAB}$ compared with the highest licensed dose of extended release tolterodine.

The duration of most clinical trials is 12 weeks, owing, among other factors, to cost constraints, intensity of surveillance, and the ethical considerations of long-term placebo treatment. Patients receive detailed follow up and incentives such as free study medication in order to complete the trial. Long term real world efficacy and persistence with treatment cannot be easily extrapolated from short-clinical trials.

Participants in a 12-week study were offered a 40-week open label extension of solifenacin at both 5 and $10 \mathrm{mg}$ doses. The aim of the study was to assess the long-term efficacy and tolerability of solifenacin (Haab et al 2005). Participants were followed up at 3 intervals. Traditional outcome measures such as frequency, urgency, urgency incontinence, and nocturia episodes per 24-hour period and volume voided per void were used. 


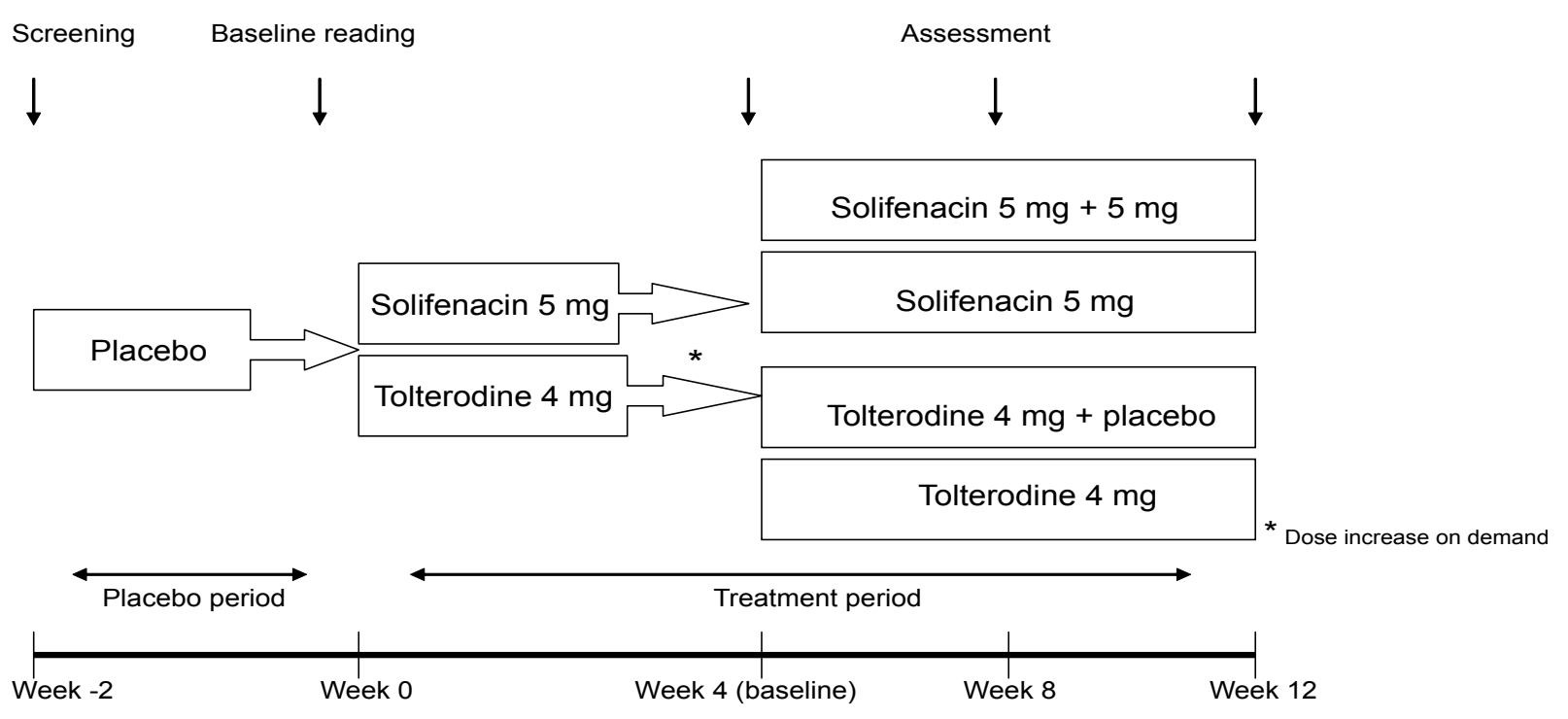

Figure 4 Design of the STAR study.

1633 patients took part in the study. $42 \%$ (685) of patients commenced and continued with a $5 \mathrm{mg}$ dose of medication. $6.6 \%$ (108) chose to increase the dose of study medication but subsequently went back to the lower dose. $51 \%$ of patients' dose escalated and continued with the $10 \mathrm{mg}$ dose of solifenacin. $81 \%$ of study participants completed the study.

Figure 5 shows the changes in bladder diary variables over the 1-year extension period. At the end of the study period, the mean number of incontinence and micturition episodes per 24 hours had decreased by $66 \%$ and $23 \%$, respectively. $58 \%$ of patients who experienced more than one incontinence episode per day at the beginning of the study were continent at the conclusion of the trial.

$4.7 \%$ of patients withdrew from treatment due to adverse events $(0.4 \%$ withdrawals were due to dry mouth). Dry mouth was reported by $10 \%$ of patients on the $5 \mathrm{mg}$ dose and $17 \%$ on the $10 \mathrm{mg}$ dose of solifenacin. The overall reported rate of dry mouth was $21 \%$. More than half of episodes of dry mouth, constipation and blurred vision were mild in severity.

At the end of the study, patients were asked about satisfaction with treatment and tolerability, $85 \%$ were satisfied, and $99 \%$ found tolerability of solifenacin to be satisfactory or acceptable.

The Kings health questionnaire (KHQ) (Kelleher et al 1997b), a 10-domain quality of life instrument designed for the assessment of quality of life among patients with lower urinary tract dysfunction, was used in 2 of the phase 3 studies (Chapple et al 2004b; Cardozo et al 2004) and the open label extension study. The domains of the KHQ assess general health perceptions, incontinence impact, role, social and physical limitations, personal relationships, sleep/energy, emotions, severity measures, and symptom severity. There are 34 linguistic validations of the KHQ and it has been used in many studies of drug therapy for OAB (Kelleher et al 2005).

Quality of life data analysis of a 12-week trial conducted by Chapple et al (2004b) showed a significant improvement in 5 of the domains of the KHQ in patients taking 5 and $10 \mathrm{mg}$ doses of solifenacin compared with placebo (role limitations, physical limitations, emotions, severity measures, and symptom severity). Patients taking $10 \mathrm{mg}$ of solifenacin also reported a significantly greater score in the incontinence impact domain. Results from the phase 3 trial study by Cardozo et al (2004) also showed statistically significant improvements in 5 domains of the KHQ for both doses of solifenacin compared to placebo (incontinence impact, role limitations, emotions, sleep/energy, and symptom severity). Patients taking the $10 \mathrm{mg}$ dose of solifenacin also reported an improvement in the physical limitations and severity measures domains (Figure 6).

Quality of life data from the long term extension study showed a significant improvement in 9 of the 10 domains of the KHQ (with the exception of the personal relationships domain). Almost two thirds of this improvement occurred in the first 3 months of treatment and was sustained for the duration of the study. Analysis of these data showed a significant improvement in all quality of life domains for patients treated with 5 and $10 \mathrm{mg}$ of solifenacin, which were $17 \%$ for the general health perceptions domain, and $35 \%-48 \%$ improvement in all other domains. Quality of life 


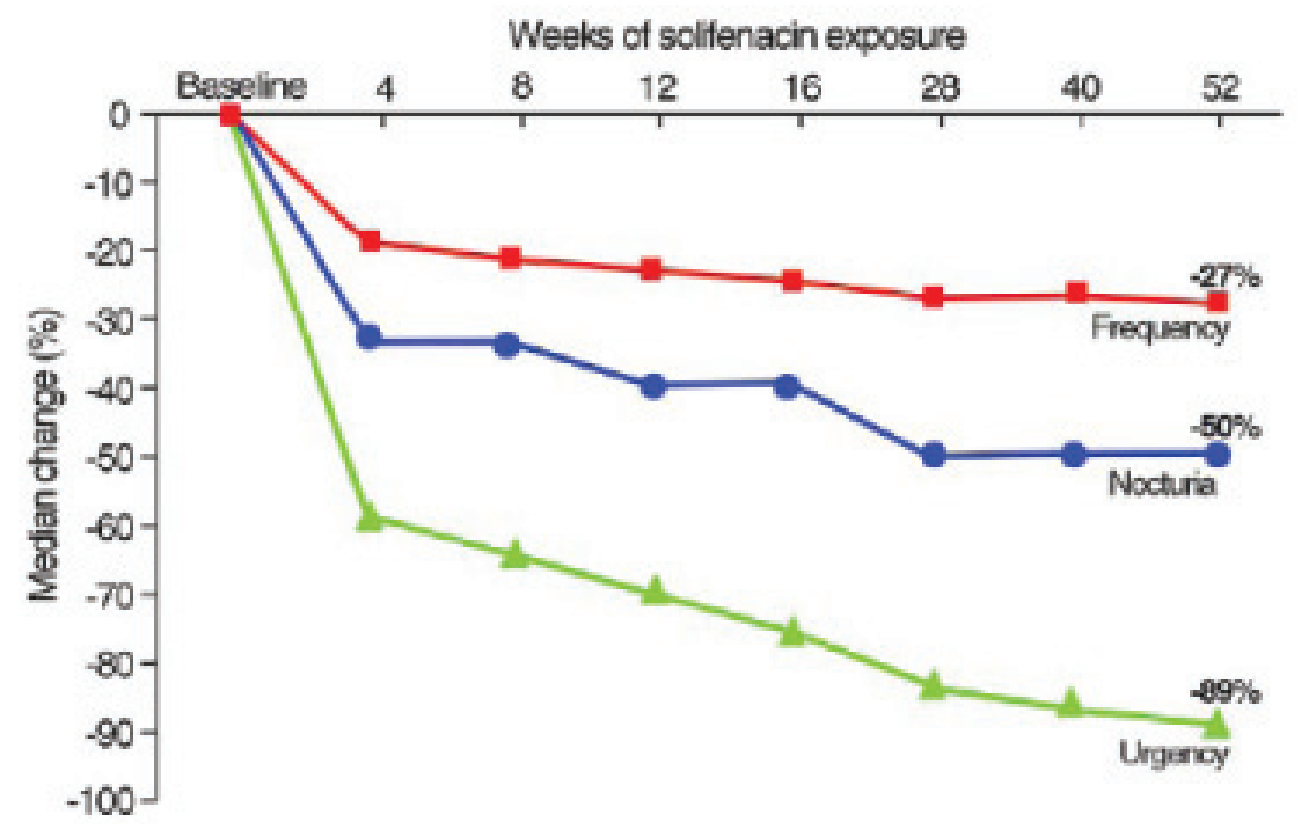

Figure 5 Median percentage reductions in frequency, urgency, and nocturia in long-term solifenacin treated patients. Reprinted with permission from Haab F, Cardozo L, Chapple C, et al. 2005. Long term open-label solifenacin treatment associated with persistence with therapy in patients with overactive bladder syndrome. Eur Urol, 47:376-84. Copyright (c) 2005 Elsevier.

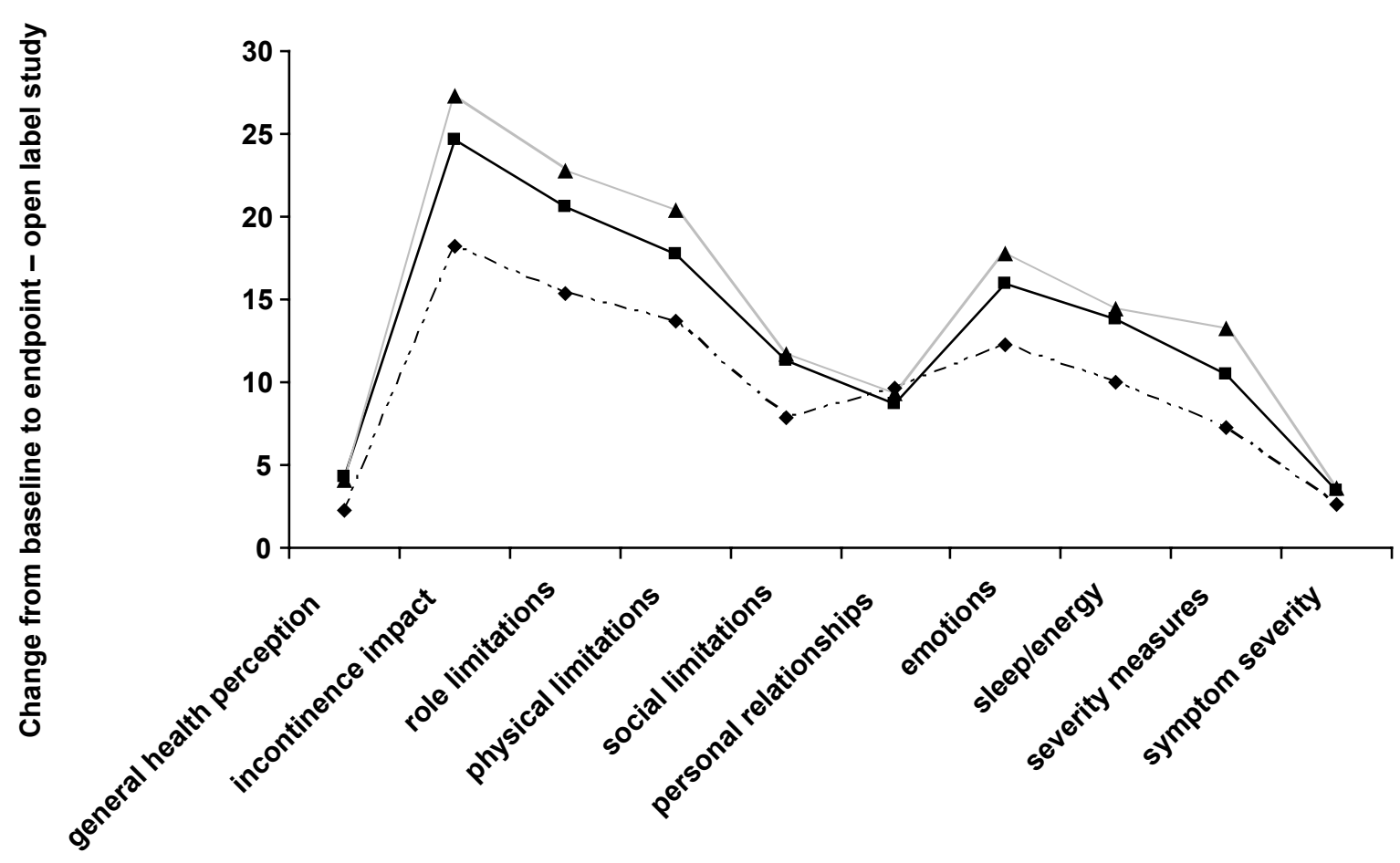

KHQ domains

\begin{tabular}{|c|c|c|}
\hline$-\bullet$ placebo & $\longrightarrow-5 \mathrm{mg}$ Solifenacin & $\triangle 10 \mathrm{mg}$ Solifenacin \\
\hline
\end{tabular}

Figure 6 Pooled changes from baseline in the KHQ domains for 2 of the phase 3 trials for solifenacin. 


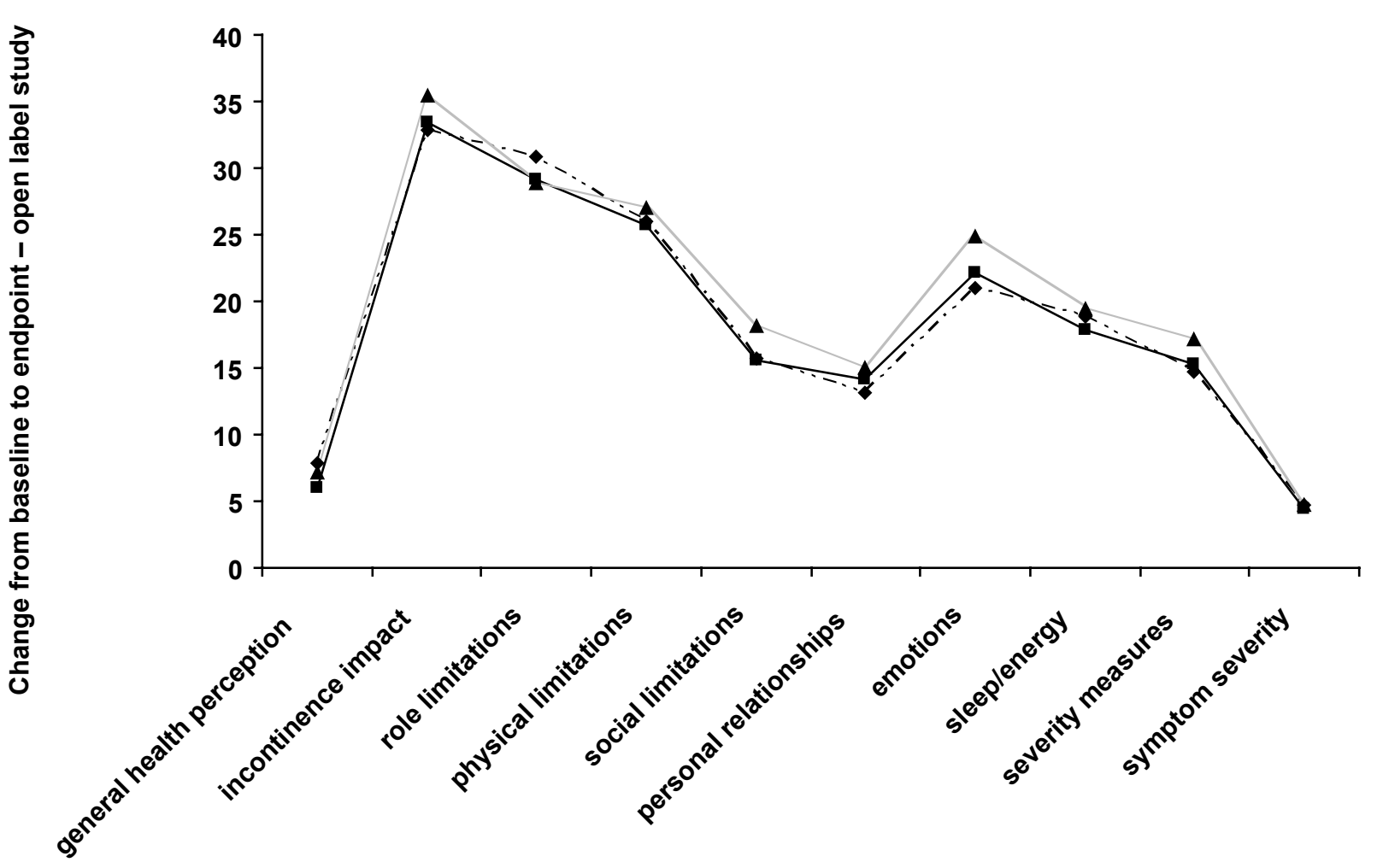

KHQ domains

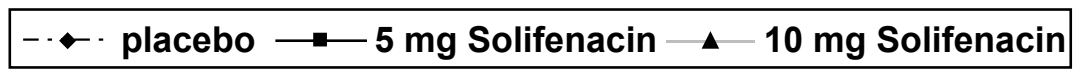

Figure 7 Changes from baseline in the KHQ domains for the open label solifenacin extension study.

improvements continued throughout the 40 -week extension period (Figure 7).

Previous studies of solifenacin have used changes in micturition frequency as the primary outcome measure of treatment effectiveness. Two recent trials have used urgency as the primary outcome variable. The Venus trial (Vesicare efficacy and safety in patients with urgency study) investigated the efficacy and safety of 5 and $10 \mathrm{mg}$ doses of solifenacin using a novel outcome measure of urgency called warning time. Warning time is defined as "the time from the first sensation of urgency to voluntary micturition or incontinence" (Chalifoux 1980). Warning time was measured using a stopwatch for a 1-day period prior to study-related visits.

739 patients participated in the study, 372 received either 5 or $10 \mathrm{mg}$ solifenacin, and 367 received placebo. The results of the study showed a significant increase in warning time in patients receiving solifenacin, 186.4 seconds versus 54.7 seconds in the placebo group. Qualitative measures of urgency were evaluated using the urgency perception scale (UPS); a validated three point scale and the Indevus urgency severity scale (IUSS); a single item patient reported measure of urgency severity (Toglia et al 2006a). Full data from this trial had not been published at the time of preparing of this manuscript. Previous clinical trials evaluating tolterodine and darifenacin have used warning time as an endpoint measure; however have failed to show a significant increase in warning time (Toglia 2006b; Zinner et al 2006).

Cardozo et al (2003) have previously shown a significant improvement in warning time for darifenacin compared to placebo in a randomized, double blind, placebo controlled study. The dose of darifenacin used was $30 \mathrm{mg}$, namely twice the maximal licensed dose for the treatment of OAB.

The 16-week Sunrise study (solifenacin in the treatment of urgency symptoms of $\mathrm{OAB}$ in a rising dose, randomized, placebo controlled, double blind, efficacy trial) assessed the effects of 5 and $10 \mathrm{mg}$ solifenacin on urgency severity and bother. The majority of clinical trials of OAB treatments use frequency and incontinence episodes as primary outcome variables as they are easy to objectively measure. Urgency is difficult to measure but remains the defining and most bothersome symptom of $\mathrm{OAB}$ and as such of greatest importance to patients and prescribers (Cardozo et al 2006a). All patients 
were offered dose escalation half way through the study, the dose of solifenacin dispensed was based on a second randomization, whereby patients taking $5 \mathrm{mg}$ solifenacin had a $50 \%$ chance of receiving a $10 \mathrm{mg}$ dose. Urgency severity and bother were chosen as the primary end points for the study. A validated 5-point urgency scale; the PIUS, and a visual analogue scale (VAS) assessing urgency bother were used. The perception of bladder condition (PBC) which is a validated, single item questionnaire, and a treatment satisfaction visual analogue scale were also used. Traditional bladder diary measurements namely, frequency, urgency and incontinence episodes per 24 hours were used as secondary outcome variables.

503 patients received solifenacin, starting at $5 \mathrm{mg}$, which could be increased to $10 \mathrm{mg}$ if required. 206 patients received placebo. Patients receiving solifenacin achieved a significantly greater reduction in urgency, frequency, and urge incontinence episodes when compared with placebo. These trends were also reflected in the qualitative measures. There was a $42.4 \%$ and $31.7 \%$ reduction in the urgency bother VAS and PBC scores respectively. Treatment satisfaction was significantly increased.

The incidences of dry mouth and constipation were higher in patients receiving solifenacin $(15.8 \%$ and $6.9 \%$ respectively) than placebo $(2.7 \%$ and $2.2 \%$ respectively). The rate of blurred vision was equivocal. Discontinuation rates for solifenacin and placebo were similar (12.1\% and 11.6\%) (Cardozo et al 2006b). Full data from this trial had not been published at the time of preparing this manuscript.

\section{Conclusion}

Solifenacin was launched in 2005 as the first once-daily, flexible-dose, M3-receptor-selective antimuscarinic for the treatment of $\mathrm{OAB}$. The efficacy and tolerability of solifenacin for the treatment of all symptoms of $\mathrm{OAB}$ has been evaluated in a number of large, placebo controlled, randomized trials. Long-term safety, efficacy, tolerability, and persistence with treatment have been established in an open label 40-week continuation study. Studies have shown that $50 \%$ of patients select the higher and lower dose of solifenacin respectively in the clinical trial setting. Higher doses of solifenacin are not associated with higher rates of discontinuation. Solifenacin has been shown to improve patients' perception of their bladder symptoms and the quality of life of OAB sufferers. In addition, recent studies utilizing an array of measures of urgency have shown that irrespective of the measure used urgency is improved by solifenacin. A large post-marketing head-to-head study comparing solifenacin flexible dose and extended release tolterodine fixed dose showed a significant benefit of solifenacin treatment.

In clinical practice, patients are commenced on $5 \mathrm{mg}$ of solifenacin and offered dose titration at 4-6 weeks of treatment. This may enhance treatment efficacy, and allow optimum control of $\mathrm{OAB}$. Whether optimal dose titration in clinical practice mirrors that seen in clinical trials is yet to be established.

\section{Conflicts of interest}

Ramandeep Basra has no conflicts of interest. Con Kelleher has acted as a consultant for Astellas, Pfizer, Allergan and Tanabe.

\section{References}

Abrams P, Kelleher CJ, Kerr LA, et al. 2000. Overactive bladder significantly affects quality of life. Am J Manag Care, 6(11 Suppl):S580-90.

1997. Birns J, Malone-Lee JG, and the Oxybutynin CR study group Controlled-release oxybutynin maintains efficacy with a $43 \%$ reduction in side effects compared to conventional treatment. Neurourol Urodyn, 16:429-30.

Braverman AS, Luthin GR, Ruggieri MR. 1998. M2 muscarinic receptor contributes to contraction of the denervated rat urinary bladder. $\mathrm{Am} \mathrm{J}$ Physiol, 275:R1654-60.

Cardozo L, Prescott K, Serdarevic D, et al. 2003. Can medication prolong warning time [poster]. Presented at ICS, Florence Italy.

Cardozo L, Lisec M, Millard R, et al. 2004. Randomized, double-blind placebo controlled trial of the once daily antimuscarinic agent solifenacin succinate in patients with overactive bladder. J Urol, 172:1919-24.

Cardozo L, Dewilde T, Feyereisl J, et al. 2006. Solifenacin significantly reduces both urgency severity and bother: Results from the flexible dose, placebo controlled, multinational Sunrise study. IUGA, Athens, Greece.

Cardozo L, Nagy G, Kiss L, et al. 2006. Solifenacin in the treatment of urgency symptoms of overactive bladder in a flexible dose, placebo controlled trial. The sunrise study IUGA, Athens, Greece.

Chalifoux P. 1980. Urinary continence/incontinence. Recognizing warning time: a critical step towards continence. Geriatr Nurs, 1:254-5.

Chapple CR. 2000. Muscarinic receptor antagonists in the treatment of overactive bladder. Urology, 55(5A Suppl):33-46.

Chapple CR, Arano P, Bosch J, et al. 2004. Solifenacin appears effective and well tolerated in patients with symptomatic idiopathic detrusor overactivity in a placebo- and tolterodine-controlled phase 2 dosefinding study. BJU Int, 93:71-7.

Chapple C, Rechberger T, Al-Shukri S, et al. 2004. Randomized, doubleblind placebo-and tolterodine-controlled trial of the once-daily antimuscarinic agent solifenacin in patients with symptomatic overactive bladder. BJU Int, 93:303-10.

Chapple CR, Martinez-Garcia R, Selvaggi L, et al. 2005. A comparison of the efficacy and tolerability of solifenacin succinate and extended release tolterodine at treating overactive bladder syndrome: results of the STAR trial. Eur Urol, 48:464-70.

Haab F, Cardozo L, Chapple C, et al. 2005. Long term open-label solifenacin treatment associated with persistence with therapy in patients with overactive bladder syndrome. Eur Urol, 47:376-84. Epub 2005 Jan 5.

Hatanaka T, Ukai M, Ohtake A, et al. 2003. In vitro tissue selectivity profile of solifenacin succinate (YM905) for urinary bladder over salivary gland in rats and monkeys [poster]. ICS, Florence Italy.

Hedge SS, Choppin A, Bonhaus D, et al. 1997. Functional role of M2 and M3 receptors in the urinary bladder of rats in vivo and in vitro. $\mathrm{Br} \mathrm{J}$ Pharmacol, 120:1409-18. 
Hedge SS, Eglen RM. 1999. Muscarinic receptor subtypes modulating smooth muscle contractility in the urinary bladder. Life Sci, 64:419-28.

Irwin D, Milsom I, Hunskaar S, et al. 2006. Population-based survey of urinary incontinence, overactive bladder, and other lower urinary tract symptoms in five countries: Results of the EPIC study. Eur Urol, 50:1306-14

Kay G, Crook T, Rekeda L, et al. 2006. Differential effects of the antimuscarinic agents darifenacin and oxybutynin ER on memory in older subjects. Eur Urol, 50:317-26. Epub Apr 19.

Kelleher C, Cardozo L, Chapple C, et al. 2005. Improved quality of life in patients with overactive bladder symptoms treated with solifenacin. BJU Int, 95:81-5.

Kelleher CJ, Cardozo LD, Khullar V, et al. 1997a. A medium term analysis of the subjective efficacy of treatment for women with detrusor instability and low bladder compliance. Br J Obstet Gynaecol, 104:988-93.

Kelleher CJ, Cardozo LD, Khullar V, et al. 1997b. A new questionnaire to assess the quality of life of urinary incontinent women. Br J Obstet Gynaecol, 104:1374-9.

Milson I, Abrams P, Cardozo L, et al. 2001. How widespread are the symptoms of overactive bladder and how are they managed? A population-based prevalence study. BJU Int, 87:760-6.

Monahan BP, Ferguson CL, Killeavy ES, et al. 1990. Torsades de pointes occurring in association with terfenadine use. JAMA, 264P:2788-90.

Ohtake A, Hanataka T, Ikeda K, et al. 2003. In vivo bladder selective profile of solifenacin succinate (YM905) over salivary gland in mice and rats [poster]. ICS Florence Italy.
Onderwater RCA. 1999. Identification of human liver CYP isoforms involved in the metabolism of YM905. Yamanouchi Europe BV, Registration number D199900072-01.00. International study ID 905-ME-020.

Ouslander JG. 2004. Management of overactive bladder. $N$ Engl J Med, 350:786-99.

Stewart WF, Van Rooyen JB, Cundiff GW, et al. 2003. Prevalence and burden of overactive bladder in the United States. World J Urol, 20:327-36.

Toglia M, Andoh M, Hussain I. 2006a. Solifenacin improves urgency symptoms as assessed by voiding diaries and patient-reported outcomes (PRO) in patients with overactive bladder [poster]. ICS, Christchurch, New Zealand.

Toglia M, Andoh M, Hussain I. 2006b. Solifenacin improved warning time significantly compared to placebo in patients with overactive bladder [poster]. IUGA, Athens, Greece.

Yamanouchi Pharma America, Inc. Nov 2004. Vesicare prescribing information [online]. Accessed 17 October 2006. URL: http://www. vesicare.com/assets/Vesicare_prescribing_info.pdf.

Zinner N, Susset J, Gittelman M, et al. 2006. Efficacy, tolerability and safety of darifenacin, an M3 selective receptor antagonist; an investigation of warning time in patients with OAB. Int J Clin Pract, 60:119-26. 